\title{
Slowing the Progression of Myopia in Children with the MiSight Contact Lens: A Narrative Review of the Evidence
}

\author{
Alicia Ruiz-Pomeda (D) · César Villa-Collar (D)
}

Received: August 25, 2020 / Published online: September 11, 2020

(C) The Author(s) 2020

\begin{abstract}
Myopia has become a major public health problem in the world due to the increase in its prevalence in the past few decades and due to sight-threatening pathologies associated with high myopia such as cataracts, glaucoma and especially myopic maculopathy. This article is a narrative review of the evidence that currently exists on a contact lenses (CLs) specifically designed to correct myopia and to slow its progression. To contextualise the topic we discuss the different classifications and definitions that have been used for myopia, the current burden of being myopic, and current treatment options to prevent and control its progression. There is evidence that exposure to sunlight reduces the risk of myopia onset and pharmacological treatment with atropine has been shown to be the most effective therapy for controlling its progression, followed by optical
\end{abstract}

Digital Features To view digital features for this article go to https://doi.org/10.6084/m9.figshare.12879014.

A. Ruiz-Pomeda

Department of Ophthalmology, Hospital

Universitario de Mostoles, Mostoles, 28935 Madrid, Spain

C. Villa-Collar $(\bowtie)$

Department of Pharmacy, Biotechnology, Nutrition and Optics and Optometry, Universidad Europea de Madrid, Villaviciosa de Odón, 28670 Madrid, Spain e-mail: villacollarc@gmail.com interventions such as CL fitting (orthokeratology or CLs specific for myopia control) designed to decrease retinal peripheral hyperopic defocus that seems to be the theory that suggests that axial elongation is driven by this defocus and explains why the eye continues to grow abnormally after emmetropisation and generates myopia. We will especially focus on MiSight CLs. MiSight is a daily replacement soft contact lens that has been clinically proven and approved by the US Food and Drug Administration (FDA) to control the progression of myopia in children. We analyse the optical design of MiSight CLs, as well as the results of the different efficacy and safety studies that led to the approval of the lens by the FDA. We also expose current knowledge gaps, limitations and future directions.

Keywords: MiSight contact lens; Myopia control; Soft contact lens for myopia control 


\section{Key Summary Points}

\section{Why carry out this study?}

Currently, myopia affects approximately $30 \%$ of the world's population and its prevalence is expected to affect $50 \%$ by 2050. Myopia has become a major public health problem in the world due to the increase in its prevalence in the past few decades and due to sight-threatening pathologies associated with high myopia such as cataracts, glaucoma and especially myopic maculopathy.

Myopia is the most common refractive error in adolescents and young adults in most parts of the world.

\section{What did the study ask?/What was the} hypothesis of the study?

Slowing the progression of myopia in children is an issue of particular interest to parents and to the scientific community. Previous studies have shown that different peripheral defocus soft contact lens designs may be effective in slowing down axial elongation and myopia progression in children compared with control subjects.

\section{What was learned from the study?}

This article is a narrative review of the evidence that exits around myopia. It discusses current treatment options to prevent and control its progression, especially focusing on MiSight contact lenses (CLs).

\section{DIGITAL FEATURES}

This article is published with digital features to facilitate understanding of the article. To view digital features for this article go to https://doi. org/10.6084/m9.figshare.12879014.

\section{INTRODUCTION}

The objective of this paper is to review the current scientific evidence on the MiSight contact lens (CLs), specifically designed to correct myopia and to slow down its progression. To contextualise the topic, we will begin with a brief introduction on basic concepts of myopia such as its definition and aetiology, the current burden in children and the current treatment options and gaps. This article is based on previously conducted studies and does not contain any studies with human participants or animals performed by any of the authors.

\section{DEFINITION AND AETIOLOGY OF MYOPIA}

Myopia has been defined and classified in a wide variety of ways such as the aetiology, age of onset, the anatomical characteristics of the eyeball, the amount of myopia (in diopters, D), progression pattern and structural complications, among others. Flitcroft et al. [1] proposed to cover five aspects of myopia: optics, diagnostic thresholds, aetiology, progression and structural complications. From an optical point of view, myopia is defined as a refractive error in which rays of light entering the eye parallel to the optic axis are brought to a focus in front of the retina when ocular accommodation is relaxed. This usually results from the eyeball being too long from front to back, but can be caused by an overly curved cornea, a lens with increased optical power or both. It is also called nearsightedness [2].

Depending on the cause, myopia is divided into axial myopia, refractive myopia or secondary myopia according to the following definitions [3]:
Axial myopia

Refractive myopia
A myopic refractive state that can be attributed to excessive axial elongation. A myopic refractive state that can be attributed to changes in the structure or location of the imageforming structures of the 
eye (i.e. the cornea and lens).

Secondary myopia A myopic refractive state for which a single specific cause (e.g. drug, corneal disease or systemic clinical syndrome) can be identified that is not a recognised population risk factor for myopia development.

The myopia to which we refer in this paper and to which the myopia control methods should be applied is axial myopia, which is also the most prevalent. By contrast, pathological myopia has an estimated overall global prevalence of $0.2-3.8 \%$ with regional variability, but varying definitions of pathological myopia used in early epidemiological studies may limit the comparability of findings [4]. The prevalence of pathological myopia-related visual impairment has been reported as $0.1-0.5 \%$ in European studies and $0.2-1.4 \%$ in Asian studies [5]. In relation to diagnostic thresholds of myopia, Flitcroft et al. define myopia as a condition in which the spherical equivalent objective refractive error is no greater than $-0.50 \mathrm{D}$ in either eye and high myopia as a condition in which the spherical equivalent objective refractive error is no greater than $-5.00 \mathrm{D}$ in either eye [6].

High myopia or pathologic myopia is also referred to as a condition with an axial length greater than 26-27 mm [7] which is usually associated with pathological axial elongation and progressive retinochoroid degeneration in the posterior pole.

It is known that myopia is a multifactorial condition that results from an interplay of environmental and genetic factors, with many variations in the prevalence, age of onset and progression among different geographic areas $[1,2]$. This gene-environment interaction is the basis of the development of the so-called simple or school myopia in which the child has completed the emmetropisation process but that, for reasons not currently known, the said process continues, giving rise to the appearance and evolution of myopia. However, pathological myopia may be present at birth or appear in the first years of life without a direct relationship with this interaction. It is in the treatment of the former, and not the latter, that myopia control methods, including the MiSight CLs, are showing evidence of efficacy [3].

It has been reported that parental myopia increases the risk for developing myopia [7]. In a meta-analysis carried out by Zhang et al. [8], it was concluded that the odds ratio (OR) of giving birth to a child with myopia, according to the prospective cohort, cross-sectional and case-control studies, was $1.53 \quad(95 \% \quad$ CI 1.21-1.85), 1.96 (95\% CI 1.53-2.39) and 2.13 (95\% CI 1.79-2.46), respectively, when one parent had myopia, and $2.10 \quad(95 \%$ CI 1.42-2.77), 2.96 (95\% CI 2.21-3.71) and 2.13 (95\% CI 1.79-2.46), respectively, when two parents had myopia.

But the rapid increase in the prevalence of myopia in the last few decades cannot be explained by genetics alone. It is known that its development is the result of the interaction between genetic and environmental risk factors [9] such as excessive close-up work, shortage of sunlight early in life, and higher level of education [10]. Myopia can also be classified by age as childhood or "school" myopia and late onset (after 15 years of age); younger age at myopia onset is the main factor that contributes to faster childhood myopia progression, this factor being independent of sex, ethnicity, time spent reading and parental myopia [11]. Once myopia has started, it keeps progressing to the late teens and early adulthood [2], although it seems that progression is greatest between ages 8 and 15 years because of continued growth of the eyeball during childhood [10]. The increase in the prevalence of high myopia is due to the increasingly early onset of myopia and its rapid progression. Myopia can be associated with an increased risk of developing ocular pathologies such as retinal detachment, cataracts, glaucoma or myopic maculopathy that may lead to vision loss and even blindness. Ocular complications due to myopia can affect different structures of the eye and present clinically with different diagnoses. 


\section{CURRENT BURDEN OF MYOPIA IN CHILDREN}

Currently, myopia affects at least $30 \%$ of the world's population, being the most common refractive error in adolescents and young adults in most parts of the world. In recent years the prevalence of myopia is increasing worldwide [12]. Recent meta-analyses [12] have suggested that close to half of the world's population may be myopic by 2050 , with as much as $10 \%$ highly myopic [13]. The increase of prevalence is higher among East Asians than similarly aged white people [12]. The increase in axial length in myopic eyes makes the retinal epithelium, choroid and sclera stretch and become thinner [2]. Although any amount of myopia can be a risk for developing sight-threatening complications, due to this stress mechanism, high myopes, especially older people, have a higher risk of developing ocular complications such as chorioretinal atrophy, foveoschisis, choroidal neovascularisation (CNV), rhegmatogenous retinal detachment, myopic maculopathy, glaucoma and cataract $[14,15]$. Each of these complications may lead to visual impairment and blindness. The greatest myopia-related cause of irreversible vision loss is myopic maculopathy that is characterized by stretched blood vessels, peripapillary atrophy, posterior staphyloma, lacquer cracks in the Bruch membrane, geographic atrophy of the retinal pigment epithelium and choroid, subretinal haemorrhages and CNV. Myopic macular degeneration causes $12.2 \%$ of visual impairment in some countries such as Japan [12]. The risk of myopic maculopathy and its impact on public health are not limited to high myopes nor older people [16], CNV is a common cause of vision loss in people of working age, often resulting in irreversible central vision loss [17]. The clinical and socioeconomic impact of myopic CNV in Asian countries is particularly significant because of the rising trend in the prevalence and severity of pathological myopia [18]. A related epidemic of high myopia is related to early onset myopia and rapid myopic progression [14]. The increase in the prevalence and severity of high myopia has a clinical and socioeconomic impact all over the world. Therefore, progressive myopia should not only be considered as a refractive error correctable by spectacles but must also be classified as a serious ocular disease that can be irreversible [2]. The burden associated with vision impairment from uncorrected myopia and with the associated vision-threatening complications have led to the need to implement strategies to control the progression of myopia in order to reduce the risk of widespread visual impairment in myopes.

Currently, slowing the progression of myopia is one of the most important goals of myopia research. It is known that the sooner myopia appears, the greater the progression will be and recent evidence indicates that the age of onset is decreasing. Parental myopia, younger age of onset, Asian ethnicity and rapid progression of myopia are considered risk factors for becoming highly myopic and should be affected for myopia control treatment [11]. Modelling predicts that a myopia control strategy that can delay myopia by $30-40 \%$ could reduce the risk of high myopia and associated ocular complications [14] e.g. slowing myopia by $1 \mathrm{D}$ should reduce the likelihood of a patient developing myopic maculopathy by $40 \%$ [16].

\section{CURRENT TREATMENT OPTIONS AND GAPS}

In order to minimise the associated ocular pathologies related to myopia, different types of treatments (optical, pharmacological, environmental or behavioural and surgical) have been studied to prevent or delay the onset of myopia and to slow its progression.

Results of several meta-analyses show that increasing time spent outdoors correlates with a decreased prevalence of myopia [19-21]. Once a child becomes myopic, his myopia progression can be significantly reduced by a range of interventions, as compared with single-vision spectacles or placebo. Low-dose atropine has been the most effective intervention for slowing down myopic progression (efficacy between $60 \%$ and $77 \%$ according to various studies), followed by orthokeratology (efficacy between 
$37 \%$ and $56 \%$ ), peripheral defocus-modifying CLs (efficacy between $25 \%$ and $79 \%$ ) and bifocal or progressive addition spectacle lenses (average efficacy 19\%) [20, 22]. Good results have recently been published with peripheral defocus ophthalmic lens (DIMS spectacle lenses). In a 2-year study, with children between 8 and 13 years old with myopia between -1 and -5 and astigmatism no greater than $1.50 \mathrm{D}$, an axial elongation of $0.21 \pm 0.02$ was found in the DIMS group and $0.55 \pm 0.02$ in the control group, with monofocal ophthalmic lenses. Myopia progressed, in the study group, $52 \%$ less in diopters and $62 \%$ less in ocular axial growth [23]. This year a multicentre clinical trial in begins China to compare DIMS with progressive ophthalmic lenses (PALs). The aim is to recruit 600 Chinese children aged 6 to 12 years old for a 3 -year follow-up with myopia between -1 and -5 and astigmatism no greater than $1.50 \mathrm{D}$ [24]. Table 1 shows the interventions that have shown efficacy controlling myopia progression in children.

Animal and human studies have shown that axial elongation is the primary factor driving myopic progression [1]. Results of the previous mentioned strategies to reduce the progression of myopia showed a clear relationship between the impact of an intervention on refraction and

Table 1 Interventions with efficacy in controlling myopia progression in children

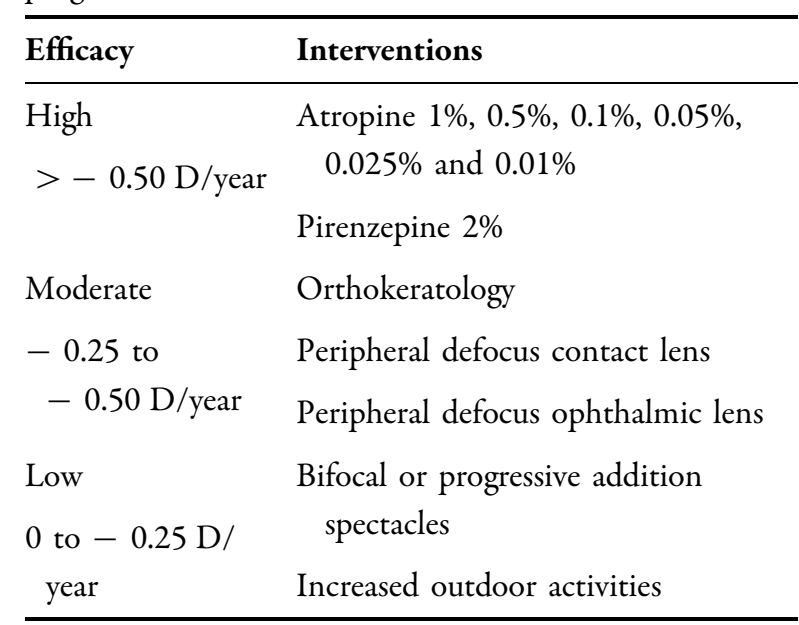

Efficacy compared with single vision spectacles as control. Own table calculated via a network meta-analysis of 30 randomised controlled trials [14]

$D$ diopters axial length. Atropine was first used to control myopia in the 1920s; since then, many studies have shown that atropine $1 \%$ can effectively control the progression of myopia but had adverse effects such as photophobia, poor near vision, dry mouth, flushing and allergies, among others [25]. Recent studies have demonstrated the safety and efficacy of atropine $0.01 \%$ and $0.05 \%$ eye drops in the prevention and treatment of myopia [25-28].

Many animal studies support the hypothesis that hyperopic defocus in the periphery causes central axial myopia [2]. This theory of the progression of myopia suggests that axial elongation is driven by peripheral retinal hyperopic defocus; in this sense, any hyperopic blur in the peripheral retina has been shown to conduct to axial length and myopia progression in animal and human studies. On the basis of this theory it has been considered that minimising the retinal peripheral hyperopic defocus or inducing peripheral myopic defocus with bifocal progressive addition spectacles (PAL), peripheral defocus ophthalmic lens [29] or with different designs of CLs could prevent myopic progression [19]. The mechanisms that support myopia control with CLs are also based on the change in retinal peripheral defocus [22]. In terms of axial length, orthokeratology (OK) and peripheral defocus-modifying contact lenses are effective in the control of myopia progression [20]. OK or corneal reshaping therapy has been demonstrated with the first type of CLs effective in slowing myopia progression. OK uses specially designed reverse geometry rigid GP lenses in overnight wear to reshape the cornea, inducing relative myopic shifts in peripheral refractive errors [30].

In addition to $\mathrm{OK}$, soft contact lenses for myopia control began to be tested in myopes. Multifocal (MF) soft contact lenses (MFSCL) are increasingly used to control myopia progression. Three different types of MFSCL for myopia control in children have been studied: bifocal concentric lenses [22, 31-34], peripheral gradient lenses [35-38] and extended depth of focus (EDOF) CLs [39]. The first two designs incorporate a central zone to correct myopic refractive error, but bifocal concentric lenses use a concentric zone of rings with plus power addition 
to simultaneously deliver peripheral myopic defocus, whereas peripheral gradient lenses simultaneously produce constant peripheral myopisation defocus that increases gradually from the central optic axis toward the periphery [22]. The third type of CL for controlling myopia progression is based on the EDOF theory. These contact lenses were designed to result in a global retinal image quality (i.e. across both the central and peripheral retina) that was improved for points on, and anterior to, the retina and degraded for points posterior to the retina to prevent axial elongation [39]. MiSight CLs would be included in the first group of bifocal or dual focus lenses based on a concentric ring design. Figure 1 shows the efficacy (expressed as a percentage) in controlling progression of myopia of the different designs of MFSCL that have been studied and that have published data.

On the other hand, the protective effect of sunlight on the growth of axial length has also been studied. Research has shown that increasing time spent outdoors correlates with a decreased prevalence of myopia [40]. Dopamine levels slow the elongation of the eyeball, while low levels are associated with an extended axial length of the eye and myopia. Many animal and human studies strongly suggest a critical role for bright light and dopamine in the modulation of eye growth [2]. Therefore, spending more time outdoors could be a complement to any other treatment to control the progression of myopia.

\section{MISIGHT CLS}

\section{Description, Specifications, Mode of Action}

MiSight $^{\circledR} 1$ day (CooperVision) is a daily replacement hydrophilic soft CL composed of Omafilcon A material that must be discarded after each removal. It has been clinically proven and FDA-approved for the correction of myopia and for slowing its progression in children with non-diseased eyes, who at the beginning of treatment are 8-12 years of age and have a refraction of -0.75 to $-4.00 \mathrm{D}$ (spherical equivalent) with no greater than $0.75 \mathrm{D}$ of astigmatism, based on clinical studies in which participants were between the ages of 8 and 12 at initial fit. Characteristics of the lens are listed in Table 2.

MiSight CLs contain a large central correction area of $3.36 \mathrm{~mm}$ surrounded by concentric zones of alternating distance and near powers which together produce two focal planes. The optical power of the correction zones corrects the refractive error while the treatment zones produce $2.00 \mathrm{D}$ of simultaneous myopic retinal defocus during both distance and near viewing (Fig. 2). The dimension of the central correction area has been designed to provide good distance visual acuity and the near power is intended as a treatment zone to prevent myopic progression [41], imposing myopic defocus on the more

\section{EFFICACY MYOPIA CONTROL (\%)}

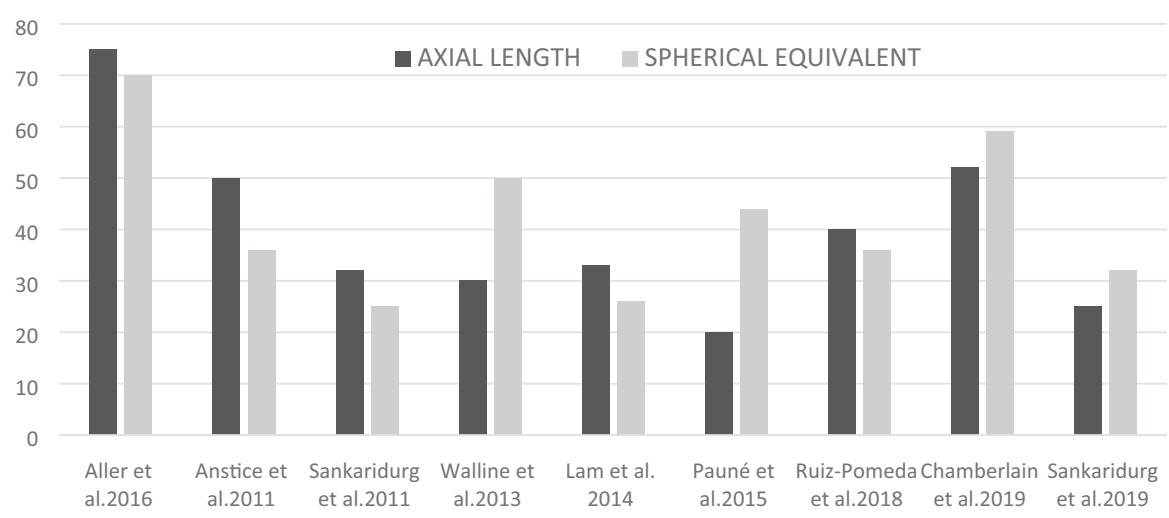

Fig. 1 Efficacy of soft contact lenses for myopia control (own figure based on data of published articles) 
Table 2 Characteristics of the MiSight lens

\begin{tabular}{ll}
\hline & Test \\
\hline Lens type & MiSight $^{\circledR}$ \\
Maker & CooperVision, Inc \\
Diameter/base curve & $14.2 / 8.7$ \\
$\quad(\mathrm{~mm})$ & \\
Contained in water & $60 \%$ \\
Ct (@ - 3.00 D) & 0.09 mm \\
Dk/t & 36.6 \\
Use regime & Daily use \\
Calendar of replacement & Daily replacement \\
Power & -0.25 to -6.00 in steps of \\
& 0.25 \\
Packaging & 30 lenses in each box \\
Dye & Light blue \\
\hline
\end{tabular}

$C t$ central thickness, $D$ diopters, $D k / t$ oxygen permeability Own table based on data of CooperVision

peripheral retina as a stimulus to slow eye growth.

Recent studies have demonstrated that MiSight CLs are effective in slowing myopia progression in children compared with control groups. The findings of a 2-year randomized clinical trial [22] showed that after 2 years of follow-up, myopia progressed slowly in the MiSight group $(0.45 \mathrm{D}$ vs $0.74 \mathrm{D}, p<0.001)$ and there was less axial elongation in the MiSight group compared to the single-vision spectacle group $(0.28 \mathrm{~mm}$ vs $0.44 \mathrm{~mm}, p<0.001)$. Chamberlain et al. [34] also demonstrated that MiSight CLs showed less refractive error change by $0.73 \mathrm{D}$ and less axial elongation by $0.32 \mathrm{~mm}$ at 36 months compared with the single-vision control CLs.

\section{Evidence that Led to FDA Approval of MiSight: Data/Studies}

MiSight approval by the FDA was based on data of efficacy from a randomized clinical trial conducted in four different countries [3] and real-world evidence. The safety and effectiveness of MiSight was studied in a 3-year randomized controlled clinical trial [34] at four investigational sites (Portugal, UK, Singapore and Canada) and also was studied in a 2-year randomized controlled clinical trial conducted in Spain [22]. These two clinical trials were designed to quantify the effectiveness of the MiSight CLs for slowing juvenile-onset myopia progression. In both cases, the primary outcomes for effectiveness were cycloplegic spherical equivalent refractive error change and axial length change over a 2-year [22] and 3-year [34] period.

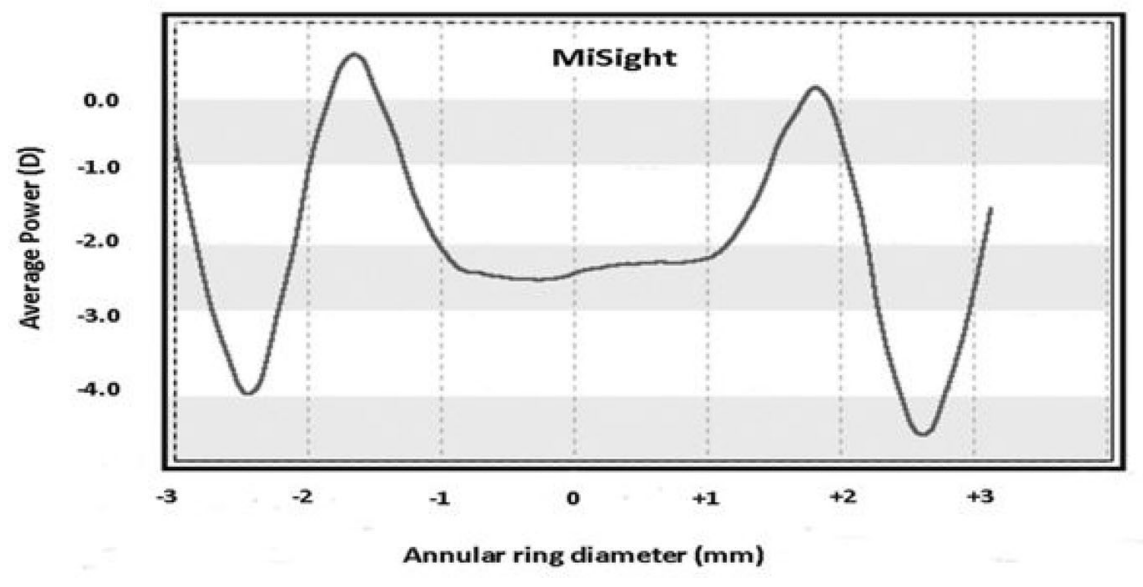

Fig. 2 Power profile of the MiSight CLs. (Ruiz-Alcocer J. Analysis of the power profile of a new soft contact lens for myopia progression. J Optom 2016;10(4):266-268.

https://doi.org/10.1016/j.optom.2016.08.003. Reproduced with permission) 
The clinical trial conducted in Spain (ClinicalTrials.gov Identifier NCT01917110) was designed to compare myopia progression in children randomized to MiSight CLs versus children corrected with single-vision spectacles (SV) over a 2-year period. In this study subjects aged 8 to 12 with myopia ( -0.75 to $-4.00 \mathrm{D}$ sphere) and astigmatism $(<-1.00 \mathrm{D}$ cylinder $)$ were also assigned to the lens study group (MiSight) or the control group (single vision). The results were published in 2018 [22]; 46 children were assigned to the MiSight group, and 33 to the single-vision spectacle group. In total, 74 children completed the clinical trial. After 2 years of follow-up, myopia and axial length progressed slowly in the MiSight group compared to the control group. Therefore, use of MiSight CLs produced lower myopia progression $(39.32 \%)$ and lower axial growth of the eye $(36.04 \%)$ at 2 years compared to spectacle use.

Better results were shown in the multicentre, parallel-group, double-masked randomized clinical trial (ClinicalTrials.gov identifier NCT01729208) with 3 years of follow-up [34]. This study enrolled children between 8 and 12 years old. From 135 subjects that received the allocated intervention (daily wear and disposable myopia control soft CLs compared with a standard daily disposable CLs), 109 subjects completed the 3-year clinical trial (53 MiSight and 56 control). Their results, which were published in August 2019 in Optometry and Vision Science, showed that the MiSight group exhibited less progression in cycloplegic spherical equivalent refraction and less progression in axial length than the control group did at each of the annual follow-up visits, representing myopia control effects of $59 \%$ and $52 \%$, respectively. Chamberlain et al. have continued the clinical trial with highly encouraging results reported for years 4 and 5, in which the original control group was refitted with MiSight ${ }^{\circledR} 1$ day. Their preliminary results for the fourth and fifth year were presented at the meeting of the American Academy of Optometry in 2016 [42] and at the BCLA Clinical Conference in 2019 [43]. In year 4 , children who were in the original control group and were refitted with MiSight CLs had a significant reduction in myopic progression for the previous single-vision 1-day wearers, indicating that MiSight ${ }^{\circledR}$ CLs are also effective when beginning myopia management at an older age.

Over the course of clinical trials by RuizPomeda and Chamberlain, there were no serious ocular adverse events in any of the study groups. Results from these studies show that correct use of MiSight CLs can be a safe option for myopia correction. The MASS study showed that the success of this treatment requires a combination of proper lens fitting, good adherence to routine follow-ups and timely treatment of complications [44].

Additionally, to estimate the rate of visionthreatening corneal infections (i.e. corneal ulcers) among children and adolescents who wear soft contact lenses daily, the FDA reviewed real-world data from a retrospective analysis of medical records of 782 children aged 8 to 12 years old from seven community eye care clinics. The results showed a rate comparable to the rate of ulcer cases among adults who wear contact lenses daily.

At present, MiSight CLs can be adapted in USA, UK, France, Spain, Portugal, the Netherlands, Belgium, Germany, Austria, Switzerland, the Nordic countries, Chile, Israel, Singapore, Malaysia, Hong Kong, Australia and New Zealand. They are not approved for sale in Japan.

In the USA, MiSight CLs are indicated for the correction of myopia and for slowing down the progression of myopia in children between 8 and 12 years with healthy eyes, with myopia between $-0.75 \mathrm{D}$ and $-4 \mathrm{D}$ and an astigmatism less than $0.75 \mathrm{D}$. The lens should be discarded after each use. In other countries the age range of application may be different.

Other aspects of MiSight CLs have been studied and published:

- MiSight CLs increased the monocular light disturbance perception compared with a single-vision spectacle correction. However, this effect decreased over the follow-up time and presented a significant binocular attenuation effect [45].

- MiSight CLs do not change the binocular and accommodative function in children wearing dual focus CLs [46]. 
- MiSight CLs wear for controlling myopia improves vision-related quality of life in children when compared with spectacle wear [47].

Other studies with MiSight CLs have been carried out by Fedtke et al. [48], Sha et al. [49], Ji et al. [50], Altoaimi et al. [51], Diec et al. [52] and Kollbaum et al. [41], showing good results regarding the performance of MiSight CLs when analysed exclusively or compared to other soft CLs for control.

\section{FUTURE PLANNED/ONGOING STUDIES IN MYOPIA CONTROL}

Some studies have studied the effects of combined treatments such as atropine and OK lenses on slowing the progression of myopia. Wan et al. [53] found improving myopia control by combining OK lenses with either $0.125 \%$ or $0.025 \%$ atropine, compared with OK lenses alone. Similar results were reported by Nozomi et al. in their 1-year follow-up study; they demonstrate that the combination of $\mathrm{OK}$ and atropine $0.01 \%$ ophthalmic solution was more effective in slowing axial elongation than $\mathrm{OK}$ monotherapy in children with myopia [54]. The Bifocal \& Atropine in Myopia (BAM) study is currently being conducted with the goal to determine whether combining $0.01 \%$ atropine and $+2.50-\mathrm{D}$ centre-distance soft bifocal contact lenses (SBCL) slows myopia progression more than SBCL alone [55]. No results have yet been published.

\section{GAPS IN OUR KNOWLEDGE, LIMITATIONS AND FUTURE DIRECTIONS}

There are some questions that need to be clarified in future studies:

1. Could there be a rebound effect when I stop wearing MiSight CLs?

2. Will MiSight CLs have the same efficacy long-term? The long-term efficacy of MiSight CLs only extends to $1-5$ years of followup. It is expected that more large-sample and long-term studies will provide strong clinical evidence in the future.

3. Will the effectiveness of MiSight CLs be the same in older children?

4. Could the efficacy of the treatment be increased by combining MiSight CLs with $0.01 \%$ atropine?

5. Which factors influence a higher or lower efficacy rate? It will also be important to investigate some aspects related to the rate of efficacy of MiSight wear. The myopia control treatment effect with MiSight CLs for an individual child may be higher or lower than the average and this may depend on many factors. Future studies will analyse which factors predispose to greater efficacy of the treatment.

\section{DISCUSSION}

The rapid increase of the prevalence of myopia and high myopia makes it a significant public health concern. High myopia $(\geq 6 \mathrm{D}$ or axial length $\geq 26$ or $27 \mathrm{~mm}$ ) increases the risk of retinal detachment and macular $\mathrm{CNV}$ among other ocular pathologies.

The objective of myopia control therapy is to slow the progression of axial length and myopia in children and thus reduce the rate of high myopia and serious associated ocular complications. It is necessary for all visual health professionals to inform society about myopia control methods that have been shown to be effective in children. It is also important to educate patients and parents on the clinical differences among treatment options available.

It has been demonstrated that atropine $0.01 \%$ is the most effective treatment for slowing myopia progression, but children who are being treated with atropine need to wear spectacles or CLs to correct their myopia during the day; for this reason, in some cases CLs wear can be preferred over the aforementioned treatment. Several studies have shown that children are sufficiently mature to safely and successfully wear different types of CLs such as daily wear rigid gas permeable CLs $[5,57]$, OK $[58,59]$ and soft CLs [60, 61]. CLs provide a constant 
treatment regardless of gaze positioning and it has been demonstrated that CLs improve vision-related quality of life in children when compared with spectacle wear [47]. On the basis of these advantages, some patients prefer other effective methods to control myopia such as OK or peripheral defocus-modifying, soft CLs. In the case of wearing OK lenses overnight, children do not need to wear CLs or spectacles in the daytime, which is a positive aspect, but on the other hand, these lenses must undergo an exhaustive use, care and cleaning routine. Several aspects to consider when choosing soft CLs for myopia control in children are safety and the level of compliance wearing CLs. Safety wearing soft CLs depends on factors such as the replacement. Most of the serious risks produced by using CLs can be prevented by following recommended wearing and using daily wear CLs [44]. It is known that daily disposable lenses are associated with a 12.5 times lower risk of corneal infiltrative events compared with reusable lenses [62]. Daily disposable MiSight CLs can be considered as a child's ideal control myopia treatment option since they do not need cleaning and disinfecting liquids or lens cases and wearing these daily CLs eliminates adverse reactions to contact lens solutions. Another important aspect to consider with soft CLs for myopia control is the level of compliance in wearing time. The effect of wearing multifocal soft CLs can be positively correlated with wearing time. A study with a novel MFSCL recommended a minimum of $5 \mathrm{~h}$ to slow myopia progression, with increasing efficacy up to $8 \mathrm{~h}$ a day of wear [33]. In general terms it could be advised to wear MFSCLs during school hours and for schoolwork at home [11]. In this sense, daily disposable CLs can provide high levels of compliance because of the simplicity of this modality of wear. To date, the only lenses approved by the FDA and the European Conformity (CE marked) for myopia control in children for daily use and replacement are MiSight CLs. Other CE-marked soft CLs for myopia control in children are Mylo (Mark'ennovy Personalized Care Ltd, Majadahonda, Spain) and Esencia ${ }^{\circledR}$ (Tiedra Farmaceutica S.L., Yecla, Spain).

\section{How Could MiSight Be Utilized in Current Management of Myopic Children: Who Will Benefit? Who Would Not Benefit?}

It is known that myopia will increase myopic children wearing traditional single-vision spectacles or contact lenses. If some design of MFSCLs were used it is expected to slow this rate of progression. According to the FDA, MiSight CLs are indicated for the correction of myopia and for slowing its progression in children who at the beginning of treatment are 8-12 years of age with healthy eyes and have a refraction of 0.75 to $-4.00 \mathrm{D}$ (spherical equivalent) with no greater than $0.75 \mathrm{D}$ of astigmatism. In this situation, MiSight CLs are expected to slow myopia progression by about $30-55 \%$.

\section{CONCLUSION}

According to current scientific evidence, MiSight contact lenses are an adequate treatment for the control of myopia in children and young adolescents. Their efficacy and safety have allowed them to obtain FDA approval and the specific CE marking for myopia control.

\section{ACKNOWLEDGEMENTS}

Funding. No funding or sponsorship was received for this study or publication of this article.

Authorship. All named authors meet the International Committee of Medical Journal Editors (ICMJE) criteria for authorship for this article, take responsibility for the integrity of the work as a whole, and have given their approval for this version to be published.

Disclosures. All authors certify that they have no affiliations with or involvement in any organization or entity with any financial interest or non-financial interest (such as personal or professional relationships, affiliations, knowledge, or beliefs) in the subject matter or materials discussed in this manuscript. The authors 
Alicia Ruiz-Pomeda and César Villa-Collar have nothing to disclose.

Compliance with Ethics Guidelines. This article is based on previously conducted studies and does not contain any studies with human participants or animals performed by any of the authors.

Open Access. This article is licensed under a Creative Commons Attribution-NonCommercial 4.0 International License, which permits any non-commercial use, sharing, adaptation, distribution and reproduction in any medium or format, as long as you give appropriate credit to the original author(s) and the source, provide a link to the Creative Commons licence, and indicate if changes were made. The images or other third party material in this article are included in the article's Creative Commons licence, unless indicated otherwise in a credit line to the material. If material is not included in the article's Creative Commons licence and your intended use is not permitted by statutory regulation or exceeds the permitted use, you will need to obtain permission directly from the copyright holder. To view a copy of this licence, visit http://creativecommons.org/licenses/by$\mathrm{nc} / 4.0 /$.

\section{REFERENCES}

1. Flitcroft DI, He M, Jonas JB, et al. IMI-defining and classifying myopia: a proposed set of standards for clinical and epidemiologic studies. Invest Ophthalmol Vis Sci. 2019;60(3):M20-30.

2. Spillmann L. Stopping the rise of myopia in Asia. Graefe's Arch Clin Exp Ophthalmol. 2020;258: 943-59.

3. Wolffsohn JS, Flitcroft DI, Gifford KL, et al. IMImyopia control reports overview and introduction. Invest Ophthalmol Vis Sci. 2019;60(3):M1-M19.

4. Wong YL, Sabanayagam C, Ding Y, et al. Prevalence, risk factors, and impact of myopic macular degeneration on visual impairment and functioning among adults in Singapore. Invest Ophthalmol Vis Sci. 2018;59(11):4603-13.
5. Wong TY, Ferreira A, Hughes R, Carter G, Mitchell $P$. Epidemiology and disease burden of pathologic myopia and myopic choroidal neovascularization: an evidence-based systematic review. Am J Ophthalmol. 2014;157(1):9-25.e12.

6. Flitcroft DI, He M, Jonas JB, et al. IMI—defining and classifying myopia: a proposed set of standards for clinical and epidemiologic studies. Invest Ophthalmol Vis Sci. 2019;60(3):M20-M30.

7. Recko M, Stahl ED. Childhood myopia: epidemiology, risk factors, and prevention. Mo Med. 2015;112(2):116-21.

8. Zhang X, Qu X, Zhou X. Association between parental myopia and the risk of myopia in a child. Exp Ther Med. 2015;9(6):2420-8.

9. Cooper J, Tkatchenko AV. A review of current concepts of the etiology and treatment of myopia. Eye Contact Lens. 2018;44(4):231-47.

10. Coviltir V, Burcel M, Cherecheanu AP, et al. Update on myopia risk factors and microenvironmental changes. J Ophthalmol. 2019;2019:4960852.

11. Gifford KL, Richdale K, Kang P, et al. IMI-clinical management guidelines report. Invest Ophthalmol Vis Sci. 2019;60(3):M184-203.

12. Holden BA, Fricke TR, Wilson DA, et al. Global prevalence of myopia and high myopia and temporal trends from 2000 through 2050. Ophthalmology. 2016;123(5):1036-42.

13. Morgan IG, French AN, Ashby RS, et al. The epidemics of myopia: aetiology and prevention. Prog Retin Eye Res. 2018;62:134-49.

14. Sankaridurg P, Conrad F, Tran H, Zhu J. Controlling progression of myopia: optical and pharmaceutical strategies. Asia-Pac J Ophthalmol (Phila). 2018;7(6): 405-14.

15. Cho BJ, Shin JY, Yu HG. Complications of pathologic myopia. Eye Contact Lens. 2016;42(1):9-15.

16. Bullimore MA, Brennan NA. Myopia control: why each diopter matters. Optom Vis Sci. 2019;96(6): 463-5.

17. Soubrane G. Choroidal neovascularization in pathologic myopia: recent developments in diagnosis and treatment. Surv Ophthalmol. 2008;53(2): 121-38.

18. Neelam K, Cheung CMG, Ohno-Matsui K, Lai TYY, Wong TY. Choroidal neovascularization in pathological myopia. Prog Retinal Eye Res. 2012;31(5): $495-525$. 
19. Weiss RS, Park S. Recent updates on myopia control: preventing progression 1 diopter at a time. Curr Opin Ophthalmol. 2019;30(4):215-9.

20. Huang J, Wen D, Wang Q, et al. Efficacy comparison of 16 interventions for myopia control in children: a network meta-analysis. Ophthalmology. 2016;123(4):697-708.

21. Xiong S, Sankaridurg P, Naduvilath T, et al. Time spent in outdoor activities in relation to myopia prevention and control: a meta-analysis and systematic review. Acta Ophthalmol. 2017;95(6): 551-66.

22. Ruiz-Pomeda A, Pérez-Sánchez B, Valls I, PrietoGarrido FL, Gutiérrez-Ortega R, Villa-Collar C. MiSight Assessment Study Spain (MASS). A 2-year randomized clinical trial. Graefes Arch Clin Exp Ophthalmol. 2018;256(5):1011-21.

23. Lam CSY, Tang WC, Tse DY, et al. Defocus incorporated multiple segments (DIMS) spectacle lenses slow myopia progression: a 2-year randomised clinical trial. Br J Ophthalmol. 2020;104(3):363-8.

24. Li Y, Fu Y, Wang K, Liu Z, Shi X, Zhao M. Evaluating the myopia progression control efficacy of defocus incorporated multiple segments (DIMS) lenses and Apollo progressive addition spectacle lenses (PALs) in 6- to 12-year-old children: study protocol for a prospective, multicenter, randomized controlled trial. Trials. 2020;21(1):279.

25. Zhao Y, Feng K, Liu RB, et al. Atropine 0.01\% eye drops slow myopia progression: a systematic review and Meta-analysis. Int J Ophthalmol. 2019;12(8): 1337-43.

26. Chia A, Chua WH, Cheung YB, et al. Atropine for the treatment of childhood myopia: safety and efficacy of $0.5 \%, 0.1 \%$, and $0.01 \%$ doses (atropine for the treatment of myopia 2). Ophthalmology. 2012;119(2):347-54.

27. Yam JC, Jiang Y, Tang SM, et al. Low-concentration atropine for myopia progression (LAMP) study: a randomized, double-blinded, placebo-controlled trial of $0.05 \%, 0.025 \%$, and $0.01 \%$ atropine eye drops in myopia control. Ophthalmology. 2019;126(1):113-24.

28. Diaz-Llopis M, Pinazo-Durán MD. Superdiluted atropine at $0.01 \%$ reduces progression in children and adolescents. A 5 year study of safety and effectiveness. Arch Soc Esp Oftalmol. 2018;93(4): 182-5.

29. Zhang HY, Lam CSY, Tang WC, Leung M, To CH. Defocus incorporated multiple segments spectacle lenses changed the relative peripheral refraction: a 2-year randomized clinical trial. Invest Ophthalmol Vis Sci. 2020;61(5):53.

30. Wildsoet CF, Chia A, Cho P, et al. IMI-interventions myopia institute: interventions for controlling myopia onset and progression report. Invest Ophthalmol Vis Sci. 2019;60(3):M106-M131131.

31. Aller TA, Liu M, Wildsoet CF. Myopia control with bifocal contact lenses: a randomized clinical trial. Optom Vis Sci. 2016;93(4):344-52.

32. Anstice NS, Phillips JR. Effect of dual-focus soft contact lens wear on axial myopia progression in children. Ophthalmology. 2011;118(6):1152-61.

33. Lam CSY, Tang WC, Tse DY-Y, Tang YY, To CH. Defocus incorporated soft contact (DISC) lens slows myopia progression in Hong Kong Chinese schoolchildren: a 2-year randomised clinical trial. Br J Ophthalmol. 2014;98(1):40-5.

34. Chamberlain P, Peixoto-De-Matos SC, Logan NS, Ngo C, Jones D, Young G. A 3-year randomized clinical trial of MiSight lenses for myopia control. Optom Vis Sci. 2019;96(8):556-67.

35. Walline JJ, Greiner KL, McVey ME, Jones-Jordan LA. Multifocal contact lens myopia control. Optom Vis Sci. 2013;90(11):1207-14.

36. Sankaridurg P, Holden B, Smith E, et al. Decrease in rate of myopia progression with a contact lens designed to reduce relative peripheral hyperopia: one-year results. Invest Ophthalmol Vis Sci. 2011;52(13):9362-7.

37. Fujikado T, Ninomiya S, Kobayashi T, Suzaki A, Nakada M, Nishida K. Effect of low-addition soft contact lenses with decentered optical design on myopia progression in children: a pilot study. Clin Ophthalmol. 2014;8:1947-56.

38. Paune J, Morales H, Armengol J, Quevedo L, FariaRibeiro M, Gonzalez-Meijome JM. Myopia control with a novel peripheral gradient soft lens and orthokeratology: a 2-year clinical trial. Biomed Res Int. 2015;2015:507572.

39. Sankaridurg P, Bakaraju RC, Naduvilath T, et al. Myopia control with novel central and peripheral plus contact lenses and extended depth of focus contact lenses: 2 year results from a randomised clinical trial. Ophthalmic Physiol Opt. 2019;39(4): 294-307.

40. Sun JT, An M, Yan XB, Li GH, Wang DB. Prevalence and related factors for myopia in school-aged children in Qingdao. J Ophthalmol. 2018;2018: 9781987. 
41. Kollbaum PS, Jansen ME, Tan J, Meyer DM, Rickert ME. Vision performance with a contact lens designed to slow myopia progression. Optom Vis Sci. 2013;90(3):205-14.

42. Chamberlain P, Back A, Lazon P, et al. 3 year effectiveness of a dual-focus 1 day soft contact lens for myopia control. Contact Lens Anterior Eye. 2018;41:S71-S7272.

43. Chamberlain P, Arumugam B, Jones D, et al. Further comparison of myopia progression in new and established myopia control treatment (MiSight $\left.{ }^{\circledR}\right)$ groups. Contact Lens Anterior Eye 2019;42(6): S1,E16.

44. Ruiz-Pomeda A, Pérez-Sánchez B, Prieto-Garrido FL, Gutiérrez-Ortega R, Villa-Collar C. MiSight Assessment Study Spain: adverse events, tear film osmolarity, and discontinuations. Eye Contact Lens. 2018;44:S180-S186186.

45. Ruiz-Pomeda A, Fernandes P, Amorim-de-Sousa A, et al. Light disturbance analysis in the controlled randomized clinical trial MiSight ${ }^{\circledR}$ Assessment Study Spain (MASS). Contact Lens Anterior Eye. 2018;42(2):200-5.

46. Ruiz-Pomeda A, Pérez-Sánchez B, Cañadas P, PrietoGarrido FL, Gutiérrez-Ortega R, Villa-Collar C. Binocular and accommodative function in the controlled randomized clinical trial MiSight ${ }^{\circledR}$ Assessment Study Spain (MASS). Graefes Arch Clin Exp Ophthalmol. 2019;257(1):207-15.

47. Pomeda AR, Pérez-Sánchez B, Cañadas Suárez MDP, Prieto Garrido FL, Gutiérrez-Ortega R, Villa-Collar C. MiSight Assessment Study Spain: a comparison of vision-related quality-of-life measures between MiSight contact lenses and single-vision spectacles. Eye Contact Lens. 2018;44:S99-104.

48. Fedtke C, Ehrmann K, Bakaraju RC. Peripheral refraction and spherical aberration profiles with single vision, bifocal and multifocal soft contact lenses. J Optom. 2019;13(1):15-28.

49. Sha J, Tilia D, Diec J, et al. Visual performance of myopia control soft contact lenses in non-presbyopic myopes. Clin Optom. 2018;10:75-86.

50. Ji Q, Yoo YS, Alam H, Yoon G. Through-focus optical characteristics of monofocal and bifocal soft contact lenses across the peripheral visual field. Ophthalmic Physiol Opt. 2018;38(3):326-36.
51. Altoaimi BH, Kollbaum P, Meyer D, Bradley A. Experimental investigation of accommodation in eyes fit with multifocal contact lenses using a clinical auto-refractor. Ophthalmic Physiol Opt. 2018;38(2):152-63.

52. Diec J, Tilia D, Thomas V, Bakaraju RC. Predicting short-term subjective vision performance of contact lenses used in myopia control. Eye Contact Lens. 2018;44(5):308-15.

53. Wan L, Wei C-C, Chen $\mathrm{C}$, et al. The synergistic effects of orthokeratology and atropine in slowing the progression of myopia. J Clin Med. 2018;7(9): 259.

54. Kinoshita N, Konno Y, Hamada N, Kanda Y, Shimmura-Tomita M, Kakehashi A. Additive effects of orthokeratology and atropine $0.01 \%$ ophthalmic solution in slowing axial elongation in children with myopia: first year results. Jpn J Ophthalmol. 2018;62(5):544-53.

55. Huang J, Mutti DO, Jones-Jordan LA, Walline JJ. Bifocal \& atropine in myopia study: baseline data and methods. Optom Vis Sci. 2019;96(5):335-44.

56. Walline JJ, Jones LA, Mutti DO, Zadnik K. A randomized trial of the effects of rigid contact lenses on myopia progression. Arch Ophthalmol. 2004;122(12):1760-6.

57. Katz J, Schein OD, Levy B, et al. A randomized trial of rigid gas permeable contact lenses to reduce progression of children's myopia. Am J Ophthalmol. 2003;136(1):82-90.

58. Walline JJ, Jones LA, Sinnott LT. Corneal reshaping and myopia progression. $\mathrm{Br} \mathrm{J}$ Ophthalmol. 2009;93(9):1181-5.

59. Cho P, Cheung SW, Edwards M. The longitudinal orthokeratology research in children (LORIC) in Hong Kong: a pilot study on refractive changes and myopic control. Curr Eye Res. 2005;30(1):71-80.

60. Walline JJ, Jones LA, Rah MJ, et al. Contact Lenses in Pediatrics (CLIP) study: chair time and ocular health. Optom Vis Sci. 2007;84(9):896-902.

61. Walline JJ, Long S, Zadnik K. Daily disposable contact lens wear in myopic children. Optom Vis Sci. 2004;81(4):255-9.

62. Efron N, Morgan PB. Rethinking contact lens aftercare. Clin Exp Optom. 2017;100(5):411-31. 